\title{
Non-Terminal Miniature Fiber Bragg Grating Temperature Probe Based in U-Shape Lossless Taper
}

\author{
D. Viegas*a,b ${ }^{a, b}$ Abad $^{\mathrm{c}, \mathrm{d}}$, J. L. Santos ${ }^{\mathrm{a}, \mathrm{b}}$, L. A. Ferreira, ${ }^{\mathrm{b}, \mathrm{c}}$, F.M. Araújo ${ }^{\mathrm{b}, \mathrm{c}}$ \\ ${ }^{\mathrm{a}}$ Faculdade de Ciências da Universidade do Porto, Rua do Campo Alegre, 687, 4169-007 Porto \\ Portugal \\ ${ }^{\mathrm{b}}$ INESC Porto, Rua Dr. Roberto Frias, 378, 4200-465 Porto, Portugal \\ ${ }^{\mathrm{c}}$ FiberSensing, Rua Vasconcelos Costa, 277, 4470-640 Maia, Portugal \\ ${ }^{\mathrm{d}}$ Universidad Europea de Madrid, C/ Tajo sn. 28670 Villaviciosa de Odón, Spain
}

\begin{abstract}
A novel miniature fiber Bragg grating (FBG) based temperature probe is presented. The sensor design integrates a ushape lossless taper thus offering the advantages of a terminal temperature probe while enabling effective serial multiplexing. We report on the experimental validation of the temperature probe design demonstrating lossless operation and effective elimination of strain cross-sensitivity.
\end{abstract}

Keywords: Temperature probe, Fiber Bragg Grating, lossless taper

\section{INTRODUCTION}

Temperature sensors based on fiber optic technology have been widely developed because of their insensitivity to electromagnetic interference and intrinsic safe operation in environments with high risk of ignition. Furthermore, large scale thermal mapping applications take advantage of the lossless remote operation and benefit from the high multiplexing capability of such technology. Different fiber optic sensor technologies have been proposed and demonstrated for temperature measurments ${ }^{1,2}$.

Over the last few years, fiber Bragg gratings ${ }^{3}$ have been used as sensing elements for a large range of applications (strain, temperature, pressure, displacement, etc.) and have been widely deployed on several areas, in particular civil engineering ${ }^{4,5}$. In fact, FBGs have rapidly become one of the most widespread temperature sensors due to their high sensitivity and multiplexing capability, compactness and reliability.

Thermal mapping applications in which size, weight and reduced cabling are key issues can greatly benefit from FBG technology. However, in such applications, FBG sensors are often placed in very small spaces where routing input and output fibers from the sensors can be a very complex task. These applications would therefore greatly benefit from temperature probe designs with leading cables exiting from a single end of the sensor enclosure. This is usually achieved by using miniature terminal probes, i.e. network designs with a single sensor per fiber lead. This approach compromises the inherent FBG multiplexing capability and turns cable routing more complex. The use of new bend insensitive fibers, allowing lossless curvature radius of $5 \mathrm{~mm}$ (Draka BendBright or Corning ClearCurve), can be helpful in this sensing networks. Yet, these fibers do not allow the implementation of a truly miniature temperature probe that enables serial multiplexing along a single fiber.

In this paper, we propose an innovative non-terminal miniature fiber Bragg grating temperature probe based in a U-shape lossless taper. This configuration consists on a FBG followed by an adiabatic fused taper that allows 180 degree lossless fiber bending with submillimeter curvature radius. The proposed design enables the implementation of miniature temperature probes with both lead fibers exiting from one end of the sensor enclosure, thus ensuring in-series lossless sensor multiplexing. The proposed design further eliminates the FBG cross-strain sensitivity, since any stress on the leading fibers is effectively transferred to the taper curvature. 


\section{EXPERIMENTAL}

\subsection{Fused Taper manufacturing}

Different tapers were manufactured in order to optimize the length $(\mathrm{L})$ and waist $(\mathrm{w})$ combinations that enable lossless bending. To manufacture adiabatic tapers ${ }^{6}$, a section of the fiber was heated by a horizontal arc-discharge while it was pulled by piezoelectric translation stages moving in opposite direction. Figure 1 shows photographs of the waist sections of some manufactured tapers with different fiber elongation. Figure 2 shows the dependence of the taper waist on the fiber elongation in the range from $4 \mathrm{~mm}$ to $42 \mathrm{~mm}$. This dependence was adjusted with the fitting $w=A e^{-L / B}$, being the coefficients $\mathrm{A}=123.96 \mu \mathrm{m}$ and $\mathrm{B}=6.69 \mathrm{~mm}$, and $w$ and $L$ are the waist and length of the taper, respectively.

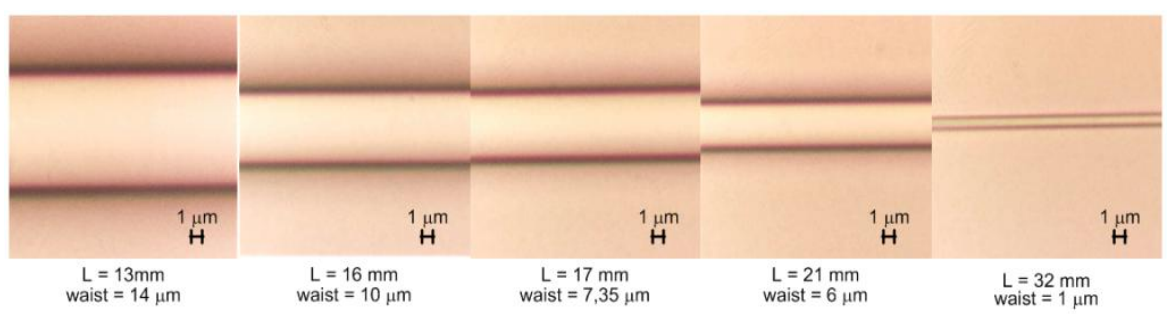

Fig. 1. Taper waist section for tapers manufactured with increasing fiber elongation.

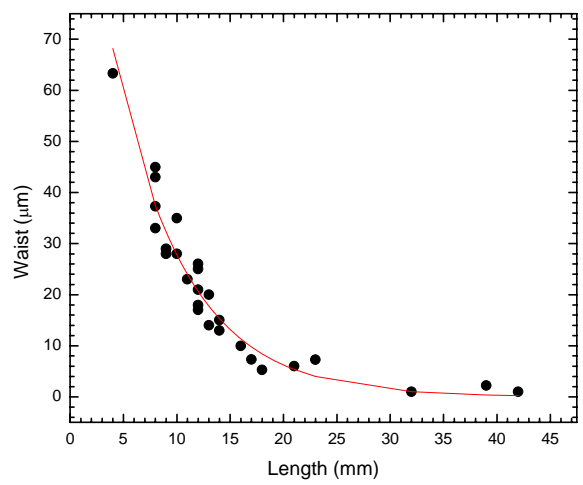

Fig. 2. Dependence of the taper waist on fiber elongation.

The manufacturing process of the tapers results in typical insertion loss below $0.5 \mathrm{~dB}$. As expected, it was readily realized that small waist sections enable bending with lower excess loss than large waist sections. So it was necessary to find a compromise between the minimum taper waist that allows lossless u-shape bending and still enables sensor miniaturization.

\subsection{Bending characterization}

An angular characterization setup was implemented to test the bending loss of the tapers (see Figure 3). The fiber was fixed in a magnetic clamp in one end while the other end was fixed to the rotation stage. The position of the taper was carefully adjusted with the rotation axis of the stage in order to minimize torsion of the taper region. It is then possible to induce controlled bending of the taper while monitoring the induced excess loss. The insertion loss was measured using a broadband source at $1550 \mathrm{~nm}$ and an optical power meter.

The dependence of the taper loss with curvature angle is depicted in Figure 4 for tapers with increasing elongation. It is possible to observe that tapers with lower elongation result in high curvature induced loss, while tapers with elongation higher than $12 \mathrm{~mm}$ enable true lossless U-shape bending. Curvature with negative angles was also induced, with similar results.

\subsection{Temperature probe implementation}

Several temperature probes were manufactured using tapers with elongation in the $10 \mathrm{~mm}$ to $12 \mathrm{~mm}$ range. This fiber elongation does not compromise sensor miniaturization and still enables low insertion loss. The temperature probes were then completed by manufacturing a FBG a few millimeters away from the taper. The sensor design used to test the 
temperature sensitivity and cross strain insensitivity is shown in Figure 5. The sensing head was fixed in a silica substrate in order to ease handling. The taper was curved in 180 deg using the setup shown in Figure 3 and then glued to the silica part. This sensing head has dimensions small enough to be inserted inside a capillary tube with diameter of only a few millimeters for the production of a practical sensor. The length of the sensing head depends mainly on the distance between the FBG and the taper, but can be as small as $2 \mathrm{~cm}$. The total insertion loss is well below $1 \mathrm{~dB}$.
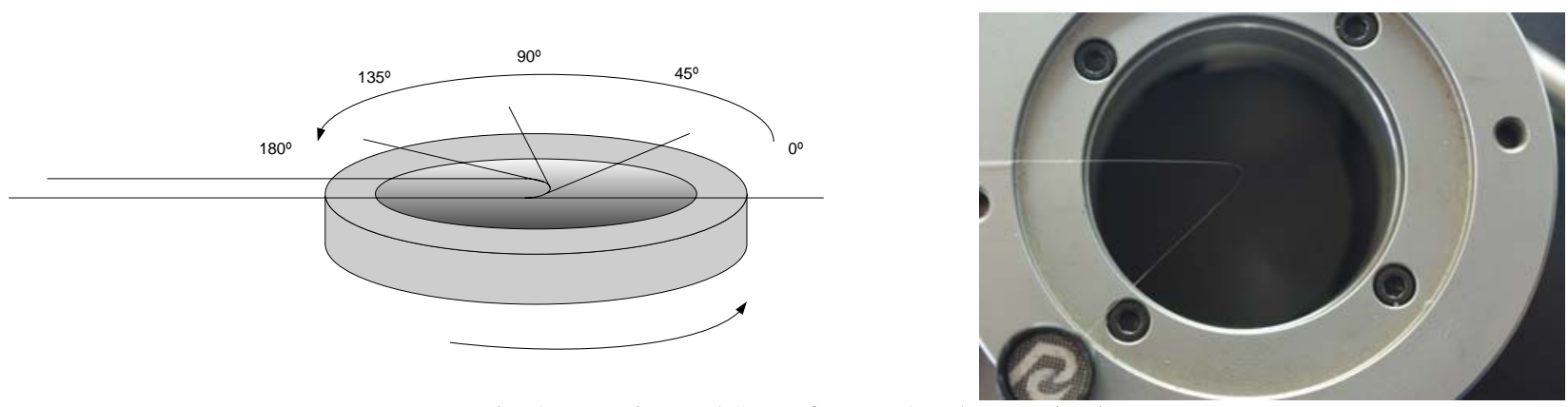

Fig. 3. Experimental Setup for angular characterization.

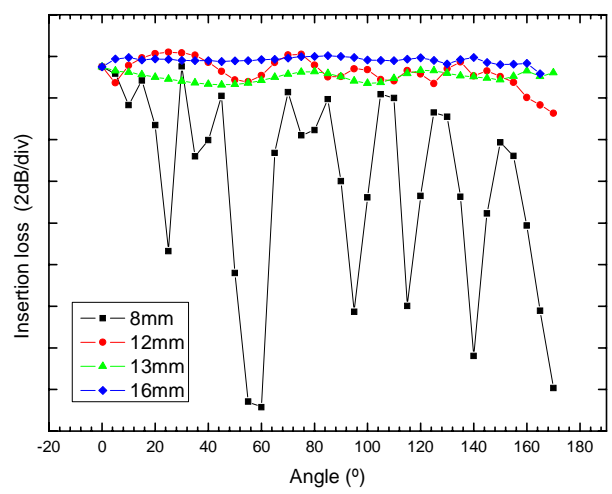

Fig. 4. Optical power loss for different curvature angles.

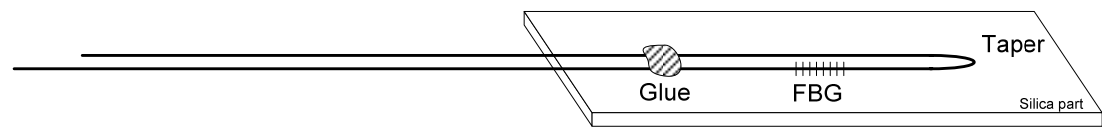

Fig. 5. Schematic of the temperature probe based on a single FBG in-line with a U-shape fused taper.

\section{RESULTS AND DISCUSSION}

\subsection{Temperature measurements}

To characterize the sensor thermal response, a full temperature cycle was made in the range of $-20^{\circ} \mathrm{C}$ to $80^{\circ} \mathrm{C}$, in 1 hour steps of $20^{\circ} \mathrm{C}$. Figure 6 shows the Bragg wavelength shift with temperature. It can be observed that the hysteresis is negligible. The data was adjusted to a second order polynomial fit, which turned out $1^{\text {st }}$ order and $2^{\text {nd }}$ order coefficients of $9.12 \mathrm{pm} /{ }^{\circ} \mathrm{C}$ and $1.09 \times 10^{-8} \mathrm{pm} /{ }^{\circ} \mathrm{C}^{2}$, respectively. These values are similar to those obtained with typical FBGs operating at the $1550 \mathrm{~nm}$ region.

\subsection{Cross-strain immunity}

To further demonstrate the temperature probe immunity to strain, the fiber leads were subject to increasing load at constant temperature $\left(\mathrm{T}=25^{\circ} \mathrm{C}\right)$. The length of the fiber under stress was $55 \mathrm{~cm}$, being the imposed displacement of the fiber leads up to $1 \mathrm{~mm}$. The maximum applied strain was in the range of $2 m \varepsilon$. Figure 7 shows the Bragg wavelength shift 
with increasing strain applied to the fiber leads. It is possible to observe that the sensor is completely immune to strain. The stress transmitted by the glue to the sensing head is transferred to the fused taper curvature which effectively isolates the FBG from any strain effect.

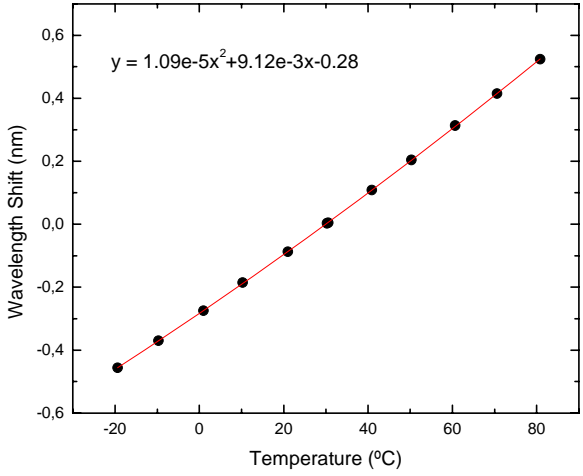

Fig. 6. Bragg wavelength shift dependence on temperature.

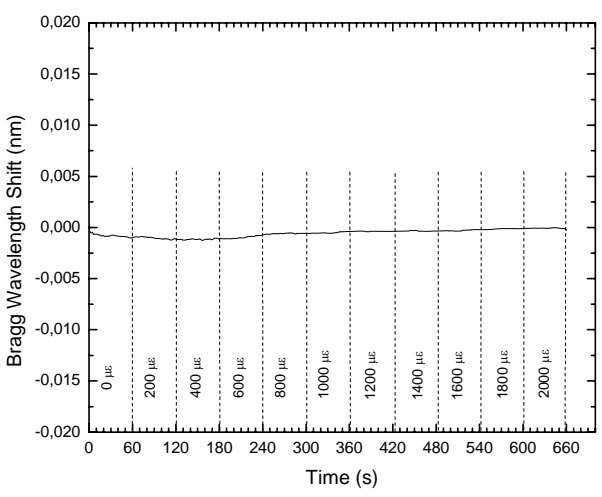

Fig. 7. Bragg wavelength shift with strain applied to the fiber leads.

\section{CONCLUSIONS}

A miniature temperature probe based on a single FBG in-line with a lossless U-shape fused taper was implemented and characterized. The proposed sensor design shows excellent thermal response, is nearly lossless and has both lead fibers exiting from the same side of the enclosure, making it an optimum candidate for large scale thermal mapping applications where a large number of sensors need to be multiplexed in-series I tiny areas. It was further demonstrated that the strain cross-sensitivity of the FBG was effectively eliminated in the proposed sensing head.

\section{ACKNOWLEDGMENTS}

This work was supported in part by Fundação para a Ciência e Tecnologia (FCT) through the project ENDURANCE Fiabilidade a Longo Prazo de Sensores de Bragg em Fibra Óptica para Monitorização Estrutural em Engenharia Civil (PTDC/ECM/77630/2006) and the grant SFRH/BD/30086/2006.

\section{REFERENCES}

1 H.Y. Choi, K.S. Park, S.J. Park, U.C. Paek, B.H. Lee, E.S. Choi., "Miniature fiber-optic high temperature sensor based on a hybrid structured Fabry-Perot interferometer”, Optics Letters, 33 (21), 2455-2457, (2008)

2 K. De Souza and T.P. Newson, "Brillouin-based fiber-optic distributed temperature sensor with optical preamplification”, Optics Letters, 25 (18), 1331-1333, ( 2000)

Y.-L. Rao, “In-fiber Bragg grating sensors” Meas. Sci. Technol. 8, 355-375, (1997)

4 A. Quintela, C. Jáuregui, J Echevarría and J M López-Higuera, “Embedded temperature-strain fibre Bragg grating sensor system validation for concrete structures”, J. Opt. A: Pure Appl. Opt. 4, S387-S390 (2002)

$5 \quad$ P. Moyo, J.M.W. Brownjohn, R. Suresh and S.C. Tjin, "Development of fiber Bragg grating sensors for monitoring civil infrastructure”, Engineering Structures, 27, 1828-1834, (2005)

6 B. P. Pal, P. R. Chaudhuri, and M. R. Shenoy, "Fabrication and modeling of fused biconical tapered fiber couplers," Fiber Integr. Opt. 22, 97-117 (2003). 